\title{
Rejouer le récit dans différentes adaptations ludiques de l'univers de J.R.R. Tolkien
}

\section{Simon Dor}

\section{OpenEdition}

\section{Journals}

Édition électronique

URL : http://journals.openedition.org/sdj/575

DOI : $10.4000 /$ sdj. 575

ISSN : 2269-2657

Éditeur

Laboratoire EXPERICE - Centre de Recherche Interuniversitaire Expérience Ressources Culturelles Education

\section{Référence électronique}

Simon Dor, «Rejouer le récit dans différentes adaptations ludiques de l'univers de J.R.R. Tolkien », Sciences du jeu [En ligne], 5 | 2016, mis en ligne le 24 février 2016, consulté le 01 mai 2019. URL http://journals.openedition.org/sdj/575; DOl : 10.4000/sdj.575

Ce document a été généré automatiquement le 1 mai 2019.

Tous droits réservés 


\title{
Rejouer le récit dans différentes adaptations ludiques de l'univers de J.R.R. Tolkien
}

\author{
Simon Dor
}

« Puisque l'histoire serait déjà connue de la plupart des joueurs mais pas de tous, le jeu devrait fonctionner et bien se jouer indépendamment de la connaissance qu'a le joueur de l'univers de

Tolkien $»^{1}$

Reiner Knizia à propos de son Lord of the Rings Board Game, cité dans Salen et Zimmerman (2004,

1 L'univers de J.R.R. Tolkien, « le plus archétypal des mondes transmédiatiques [transmedial worlds]» (Lisbeth et Tosca, 2004, p. 413), est probablement l'un de ceux qui a influencé le plus le jeu vidéo et le jeu traditionnel. Complexe et jouant sur plusieurs fronts, la Terre du Milieu est l'un de ces nombreux univers se déployant dans différents médias qui semblent avoir une propension à susciter un fort sentiment d'immersion, que ce soit par le biais de mécaniques de wargame, de jeu d'action, de jeu de stratégie ou de jeu de rôle. Le récit de la Guerre de l'anneau en est sans surprise le récit le plus adapté en jeu, en grande partie parce qu'il est le plus connu. Mais la connaissance préalable d'un récit mène très certainement à une expérience ludique bien différente de celle d'un récit inconnu. L'immersion dans la Terre du Milieu et le sentiment de présence est a priori contradictoire avec la volonté de revoir des événements qui nous ont marqués dans une expérience littéraire ou filmique. Incarner un personnage dans un univers diégétique implique qu'on cherche à se mettre à sa place, à vivre son incertitude quant aux événements futurs. Plus encore, comme le note Henry Jenkins, « nous connaissons l'histoire avant même d'acheter le jeu et serions frustrés si tout ce qu'il nous offrait était de nous recracher l'expérience originale du film » (2004). L'intérêt de rejouer le récit est certainement autre chose que celui de vivre la même expérience narrative que celle du film en sachant d'avance les événements de l'histoire. 
2 Le plaisir qui semble le plus évident est celui d'être en mesure de s'imprégner de quelque chose de l'univers dans lequel on joue, un «aura» ou un «esprit» du monde transmédiatique. Nombre d'adaptations ludiques, comme on le verra, s'en tiennent d'ailleurs à reprendre des éléments du monde sans nécessairement chercher à reprendre l'histoire elle-même, alors que d'autres permettent au joueur de jouer directement dans le contexte de la Guerre de l'anneau. On peut constater que les jeux vidéo qui adaptent la Terre du Milieu vont très souvent modifier les règles de cet univers pour reprendre des mécaniques de jeu héritées de la tradition du genre vidéoludique dans lequel ils s'inscrivent. C'est tout le contraire du jeu de plateau La Guerra dell'Anello (2004), proposé par Roberto Di Meglio, Marco Maggi et Francesco Nepitello, qui en adapte l'esprit plutôt que la lettre. Ce jeu traditionnel se centre d'une manière plus précise sur le sentiment d'incertitude des personnages. Mon texte propose une analyse comparative de différentes stratégies de représentation d'un univers fictionnel, entre jeux traditionnels et jeux numériques, dans un contexte où un joueur ou une joueuse en connaît déjà les enjeux narratifs majeurs.

C'est une conception non-instrumentale d'un média (Cisneros, 2000, p.10) et une narratologie de l'expression (Gaudreault, 1999, p. 47) que je propose ici, c'est-à-dire que le rôle du contexte narratif des jeux tiendra compte du média qui narre - en particulier en ce qui a trait aux conventions de genres. J'analyserai différents jeux en fonction de l'économie de l'information qui y est impliquée et m'intéresserai aux répercussions de la connaissance préalable de l'histoire et des conventions de genre sur les décisions que le joueur doit prendre. Ces répercussions sont-elles différentes selon qu'on soit dans un contexte de jeu traditionnel ou de jeu numérique?

4 Mon article n'a pas la prétention de faire un travail exhaustif de toutes les adaptations de Tolkien en jeu vidéo ou en jeu de société : il propose plutôt un survol de différentes stratégies d'adaptation d'un univers pour mieux comprendre le rôle des conventions de genre dans l'expression ludique. Ce qui apparaît comme un travail de comparaison entre deux médias, ici le jeu vidéo et le jeu traditionnel, doit être vu comme un essai d'une compréhension plus extensive non pas de ce que chacun des médias peut faire, mais de ce que chacun des médias fait.

5 Il s'agit au fond d'une réflexion préliminaire à une analyse comparée du rôle de la convention dans les contextes des industries du jeu vidéo et du jeu de société traditionnel. L'approche préconisée ici est celle d'une analyse textuelle, ou close-reading, telle que décrite par Jim Bizzocchi et Joshua Tanenbaum (2011). Je m'intéresserai alors à reconstruire une expérience du jeu, un certain type de lecture, à partir de ma propre expérience et de sources externes (Aarseth, 2003, p. 3). Bien que je décrirai plusieurs jeux reprenant l'univers de Tolkien, je décortiquerai plus particulièrement le jeu de stratégie en temps réel (STR) The Lord of the Rings: The Battle for Middle-earth (EA Los Angeles, 2004) - notamment sur sa réception critique - et le jeu de société La Guerra dell'Anello.

\section{Revivre l'histoire et le déroulement des événements}

6 L'enjeu central de ma réflexion, c'est l'idée que le joueur type de n'importe quel jeu qui adapte l'univers de Lord of the Rings est un joueur qui connaît d'avance le monde du jeu et les événements qui s'y dérouleront. Cette connaissance préalable du récit module nécessairement son expérience, en particulier lorsqu'il va pouvoir rejouer une histoire 
qui l'a introduit préalablement à cet univers même. Le joueur qui se ré-immerge dans un univers fictionnel qu'il a déjà connu et qui incarne des personnages dont il connaît en grande partie d'avance les destins vit un sentiment très différent de celui qui ne connaîtrait pas d'avance les enjeux de ses choix.

7 Le cas de la Guerre de l'anneau est particulièrement emblématique dans le monde de Tolkien. Sauron est le Seigneur des Ténèbres qui jadis possédait des pouvoirs particulièrement grands qu'il a consolidés dans l'anneau unique, anneau qu'il a perdu et qu'il cherche dorénavant à retrouver dans l'optique de prendre possession de la Terre du Milieu. Les serviteurs de Sauron sont pour la plupart des Orcs, soit des créatures humanoïdes sombres et corrompues, mais aussi des spectres et des créatures diverses. L'anneau a par contre été trouvé par Bilbon le hobbit, une créature de taille mi-humaine qui a la réputation de ne pas se soucier des affaires politiques et des grands enjeux de son monde. Frodon, neveu et héritier de Bilbon, a maintenant la tâche de détruire l'anneau unique dans le feu de la Montagne du Destin où il a été forgé, au fin fond du Mordor, terre désertique où résident Sauron et ses armées. Il sera aidé d'une communauté composée notamment du magicien Gandalf et du rôdeur Aragorn, héritier au trône du royaume du Gondor, l'un des peuples libres résistant à Sauron.

Entre la communauté de l'anneau et les peuples libres, d'un côté, et Sauron et ses alliés de l'autre, il y a peu de nuance morale. L'enjeu de Sauron est de retrouver l'anneau, sans qu'il ne sache tout à fait où celui-ci se trouve ni qui l'a en sa possession. Sauron envoie quelques-uns de ses plus fidèles serviteurs pour retrouver l'anneau tout en projetant d'envahir la Terre du Milieu avec ses armées. L'enjeu des peuples libres est de détruire l'anneau en l'envoyant au sein des terres ennemies, tout en défendant les terres pas encore occupées par les armées ténébreuses. Le rapport de force semble complètement déséquilibré : Sauron a une armée unie, forte, qui se bat contre des armées fragiles et fragmentées qui n'ont pas de stratégie commune. Frodon voyagera au final jusqu'au Mordor uniquement accompagné de son fidèle ami Sam, sans réel espoir de pouvoir détruire l'anneau, tandis que Sauron sous-estimera tout au long du récit la possibilité qu'ont ses ennemis à le faire.

9 Or, le joueur qui connaît d'avance l'histoire sait bien qu'il est possible (et même très probable) que l'anneau puisse être détruit. Toute l'incertitude de la quête elle-même ne peut être vécue de la même manière pour le joueur qui la connaît d'avance. Si le premier lecteur de Lord of the Rings sait aussi qu'il est très vraisemblable que l'anneau se fasse détruire, parce qu'il lit un roman qui répond aussi à un certain nombre de conventions incluant celle qui veut que le Bien triomphe généralement sur le Mal -, il n'agit pas sur le monde fictionnel même. En lisant un livre, le lecteur n'a pas à prendre de décisions à la place des personnages. Ces enjeux sont donc centraux pour un joueur davantage que pour un lecteur ou un spectateur, en particulier bien sûr lorsqu'on laisse au joueur la possibilité de changer le cours des événements. ${ }^{2}$

\section{Raconter l'histoire de manière classique}

10 La stratégie la plus simple pour préserver le récit dans un jeu est, justement, de ne pas permettre au joueur de l'influencer, en le racontant à la manière du cinéma ou de la littérature. Plutôt que de faire interagir le joueur avec le récit et de lui permettre de prendre des décisions pour ses principaux agents, le joueur va atteindre un certain nombre d'objectifs de mission pour progresser dans l'histoire. La plupart du temps, il y 
aura différents niveaux ou scénarios qu'on devra accomplir dans un certain ordre. Comme le rappelle Jonathan Lessard en parlant du jeu d'aventure The Hobbit (Beam Software, 1982) adapté du livre du même nom de Tolkien, « on s'attend à y retrouver non seulement les personnages et les lieux, mais aussi les principales péripéties du roman, préférablement dans l'ordre » (2013, p. 81). Les jeux de progression peuvent relativement facilement adapter une histoire tirée de la littérature en proposant un parcours à explorer dans un ordre fixe, où échouer à faire dérouler l'histoire correctement mène à un retour en arrière dans la fiction. Pour reprendre le vocabulaire de Marie-Laure Ryan (2001), le joueur n'est pas réellement en mode d'interactivité ontologique dans la plupart de ces expériences: toute modification au monde du jeu qui entrainerait des répercussions autres que celles prévues par l'histoire originale entraîne un échec pour le joueur, qui doit réessayer jusqu'à la réussite s'il souhaite progresser dans le jeu.

11 The Lord of the Rings: The Return of the King (Electronic Arts Redwood Shores Studio, 2003), un jeu d'action hack and slash, ${ }^{3}$ semble vouloir s'en tenir le plus possible au récit du film au sein des cinématiques, de sorte de pouvoir raconter essentiellement l'histoire telle qu'elle a été présentée dans les films (Wallin, 2007). Dans le même esprit, le mode campagne de The Battle for Middle-earth raconte l'histoire d'une manière assez fidèle à l'adaptation filmique. Lorsqu'une séquence de jeu particulière dans la campagne évoque une séquence des films, on utilise très souvent des extraits du film lui-même, présentés dans un coin de l'interface. Les personnages principaux des films ont été adaptés dans le STR (jeu en stratégie en temps réel) en "héros", soit des unités militaires uniques avec des statistiques plus élevées que l'habitude qui ont leurs pouvoirs spéciaux et qui peuvent accroître ceux-ci en accumulant des points d'expérience. ${ }^{4}$ Si dans la plupart des STR, s'assurer que ses héros survivent est une condition à ce qu'une mission réussisse, c'est pour permettre à l'histoire de suivre son cours avec ses protagonistes principaux. Dans The Battle for Middle-Earth, la mort de certains héros n'entraîne pas toujours une défaite pour le joueur : ainsi, Aragorn peut mourir dans certaines missions (Ocampo, 2004). ${ }^{5} \mathrm{Par}$ contre, au moment où l'histoire implique qu'il soit présent, Aragorn revient pour accomplir son rôle. Raconter une histoire semblable à celle du livre ou des films reste l'enjeu central. ${ }^{6}$ Comme le résume bien le critique John Keefer, «bien qu'on reste fidèle aux films, cela tend à enlever le sentiment d'urgence qu'il y a à les protéger » (2004).

Pour reprendre le terme de Lisbeth Klastrup et Susana Tosca (2004, p. 412), plusieurs jeux adaptés du monde de la Terre du Milieu n'en adaptent pas son "ethos ", soit la manière dont les personnages sont censés agir dans ce monde. L'exemple de The Lord of the Rings: The Fellowship of the Rings (Surreal Software, 2002) qu'ils donnent le montre tout à fait (p. 413). Dans une quête où Aragorn doit chercher le hobbit Merry à Bree, il peut éviter de se faire éliminer par ses ennemis s'il s'assure que les autres hobbits leur bloquent le passage : puisqu'ils sont des personnages non-joueurs, ces derniers ne peuvent se faire tuer. Placer les hobbits devant soi alors qu'il devrait les protéger est tout sauf représentatif de l'héroïsme attribué au personnage. Ce sentiment de protection des personnages se déploiera plus clairement comme on le verra dans La Guerra dell'Anello.

\section{L'esthétique du jeu classique}

13 Le plus souvent, le jeu ne respecte pas à la lettre l'histoire telle qu'elle est présentée dans les livres ou les films. Alexis Blanchet (2008, p. 74) note bien que le jeu vidéo va souvent adapter certaines scènes pour leur ajouter des défis ludiques, par exemple, en dilatant les 
séquences de combat, voire même tout simplement en ajoutant des séquences créées de toutes pièces pour l'adaptation. Battle for Middle-Earth va dilater la séquence des mines de la Moria pour y ajouter davantage d'affrontements avec Gobelins et Trolls. C'est aussi ce que Mark Rowell Wallin (2007) décrit dans son analyse des adaptations de Lord of the Rings. Dans la première séquence du jeu The Return of the King, Aragorn, Legolas et Gimli explorent les cavernes des morts-vivants pour leur demander de se battre pour eux ; or, dès l'entrée dans le tunnel, les trois protagonistes combattent des fantômes, alors que ni le film ni le livre n'ont présenté un seul combat dans les scènes et chapitres correspondants. Chaque niveau du jeu aura des puzzles à résoudre et des ennemis à affronter, ainsi qu'un boss final qui offre un défi supplémentaire (Wallin, 2007). Les séquences qui contiennent tous les éléments typiques d'un niveau de jeu - notamment celles qui exploitent la spatialité, pour reprendre l'idée d'Henry Jenkins (2004) - semblent tout indiquées pour être adaptées en y ajoutant les conventions des STR et du hack and slash, respectivement. De la même manière, le jeu d'action The Lord of the Rings: Conquest (Pandemic Studios, 2009) ajoute un niveau à Minas Morgul pour diversifier ses combats, même si aucune bataille de grande envergure ne s'y est déroulée dans le roman ou dans les films.

C'est que ces jeux s'inscrivent parfaitement dans la logique de leur genre vidéoludique. La trilogie filmique The Lord of the Rings (Peter Jackson, 2001-2003) s'inscrit dans le film épique et dans le cinéma hollywoodien populaire dans la même mesure où Battle for Middle-Earth s'inscrit dans le jeu de stratégie en temps réel: les deux convoquent des "stratégies préfabriquées pour la lecture", pour reprendre l'expression de Peter J. Rabinowitz (1987, p. 177) à propos de la littérature de genre. Parce qu'on connaît le genre, ses conventions et son contexte de production (hollywoodien), on sait d'avance que les Hobbits finiront par détruire l'anneau, on sait d'avance que le roi finira par être couronné à Minas Tirith. Le lecteur (ou spectateur) sait reconnaître des éléments qu'il a déjà lu et qui font partie d'un pattern émergent qu'il a reconnu (Rabinowitz, 1987, p. 45).

C'est essentiellement de la même manière que le joueur de jeu vidéo structure sa connaissance. Les chercheurs en psychologie cognitive se sont beaucoup intéressés aux échecs et ont constaté que la mémoire y jouait un rôle prépondérant par opposition à l'élaboration ex nihilo d'une stratégie. Adriaan de Groot note que les joueurs d'échecs expérimentés peuvent inférer des actions futures dès la stratégie d'ouverture: "quelqu'un peut reconnaître le type d'ouverture à partir de l'ensemble des pièces et voir immédiatement ce qui se passe et ce qui devrait, en principe, arriver à partir de configurations typiques des pièces - connues par son expérience du jeu " (cité dans Holding, 1985, p. 74). C'est que le joueur développe progressivement une compétence encyclopédique, pour reprendre le concept d'Umberto Eco $(1985$, p. 15) : il accumule des possibilités de jeu et leur attribue une certaine probabilité dans différents contextes. En bref, il crée dans son esprit une habitude, au sens de Charles S. Peirce (1991, p. 76), c'est-àdire une propension à faire une action particulière à différentes occasions qui apparaissent comme des occurrences d'un événement général. Eco en cite une définition plus générale: l'habitude est la «tendance [qu'a une chose] à agir de façon semblable dans des circonstances semblables dans le futur " (1985, p. 49). En demandant au joueur d'entreprendre un certain nombre d'actions dans un certain nombre de circonstances similaires, les jeux vidéo proposent au joueur de développer des habitudes stratégiques : répondre d'une manière semblable à des circonstances de jeu semblables. ${ }^{7}$ D'un jeu vidéo à l'autre au sein d'un même genre, le joueur s'attend à ce que ses habitudes stratégiques 
ne soient pas trop déstabilisées, lui permettant alors d'apprendre plus rapidement comment jouer. Le rôle de l'habitude dans l'expérience vidéoludique est vraisemblablement plus central que dans l'expérience ludique traditionnelle.

C'est sous l'égide de ces habitudes que les jeux vidéo modifient des univers fictionnels tels qu'ils existent dans d'autres médias. Battle for Middle-Earth, Conquest et The Return of the King racontent une histoire d'une façon classique en passant par une esthétique ludique tout aussi classique, chacun dans son genre. De ce déroulement du récit semble émerger l'idée que le jeu n'est qu'accessoire, qu'il n'est que prétexte à faire dérouler le monde luimême.

\section{Le monde comme thème}

Jesper Juul (2005, p. 189) a déjà suggéré que le jeu vidéo pouvait être "thématisable », c'est-à-dire que le thème d'un jeu était plus ou moins interchangeable sans que l'expérience ludique n'en soit affectée. C'est l'impression que donne d'emblée un jeu comme Guardians of Middle-earth (Monolith Productions, 2012), un Multiplayer Online Battle Arena (MOBA) se déroulant lui aussi dans l'univers de Tolkien. Il n'y a aucune justification fictionnelle à ce que Frodon, Gandalf et Sauron puissent se déplacer dans l'espace d'une arène de jeu et aient chacun des habiletés ayant relativement la même portée et la même puissance sur un champ de bataille. Deux joueurs adverses peuvent par ailleurs incarner le même personnage au détriment de la cohérence de l'univers. Guardians of Middle-earth apparaît bien davantage comme un MOBA ayant pour thème le Seigneur des anneaux que comme un jeu mettant en scène un récit se déroulant sur la Terre du Milieu.

Sans être aussi extrêmes, force est de constater que plusieurs jeux se déroulant sur la Terre du Milieu peuvent être vus jusqu'à un certain point comme des jeux «à thématiques ». The Lord of the Rings: The Third Age (Electronic Arts Redwood Shores Studio, 2004) propose dans cette lignée une distorsion de l'univers pour le moins étrange ${ }^{8}$ : le joueur incarne, à la manière d'un jeu de rôle japonais, un groupe de personnages créés spécialement pour le jeu qui ont des pouvoirs surnaturels et qui suivent les aventures des personnages principaux. Le principe des combats reprend les mécaniques de Final Fantasy $X$ (Square, 2001), en intégrant des habiletés spéciales thématisées à l'univers de Tolkien. «Fangorn Roots », par exemple, est un sort utilisable par le Ranger qui immobilise un adversaire avec des racines semblables aux Huorns de la forêt de Fangorn; "Water Stallion » est un sort de l'Elfe qui invoque un cheval d'eau à la manière dont l'a fait Arwen dans le premier film de Peter Jackson (The Fellowship of the Ring, 2001). Les principaux personnages ont été spécifiquement créés pour le jeu et ne trouvent aucun écho ni dans les films, ni dans le livre, bien qu'ils puissent affronter des personnages emblématiques de ceux-ci, notamment le Balrog aux côtés de Gandalf. Plutôt que de devoir laisser Frodon détruire l'anneau, l'adversaire ultime sera l'œil de Sauron lui-même - le jeu devant visiblement avoir un boss final pour suivre les conventions d'un jeu de rôle japonais. La Terre du Milieu semble un décor pour faire fonctionner les principes de jeu du genre.

Plusieurs critiques de Battle for Middle-Earth semblent lucides quant au rapport qu'il entretient avec l'univers de Tolkien: le jeu est pour eux une manière de vivre l'atmosphère de l'œuvre plutôt que d'en être une bonne adaptation. D'un côté, on note que le jeu est "accessible au plus grand nombre » (Superpanda, 2004), cohérent avec le style des films de Peter Jackson (Adams, 2004, p. 1), qu'il plaira aux joueurs occasionnels 
(Joe_Dodson, 2004) et aux « casual fans » de STR en ce sens que tout mise sur l'efficacité (Tucker, s.d.). Le joueur de STR plus aguerri semble en sortir moins comblé. De la même manière, on note qu'il est une bonne recréation jouable du travail de Tolkien (Keefer, 2004), que les fans devraient apprécier (Joe_Dodson, 2004). Il reste que les parties personnalisées peuvent rendre l'univers plus difficile à accepter, en particulier pour le puriste : deux factions du Gondor peuvent s'affronter entre elles, voire, comme pour Guardians of Middle-Earth, deux versions alternatives d'un même personnage. Plusieurs éléments « feront tiquer les spécialistes » (Genseric, s.d.) ; l'aura de la Terre du Milieu s'y sera perdue, même s'il y a certainement une intensité unique à jouer à un STR avec ses personnages emblématiques (Keefer, 2004).

20 Cette fonction thématique se fait aussi ressentir dans les jeux traditionnels. Risk: The Lord of the Rings (Stephen Baker et Jean-René Vernes, 2002) reprend dans les faits les mêmes règles que le Risk original (Albert Lamorisse et Jean-René Vernes, 1959), à quelques exceptions. Dans les règles de base, le joueur peut incarner ou bien une armée des peuples libres, ou bien une armée de l'ombre, sans que cela n'ait d'impact sur la jouabilité : chaque joueur joue pour lui-même contre les autres. ${ }^{9}$ L'anneau y a été adapté, en minimisant son rôle fictionnel: son parcours est la limite de temps du jeu. Une fois l'anneau détruit, la partie est terminée et le joueur qui contrôle le plus de territoires a gagné. Pour mieux faire vivre les événements fictionnels du récit, certains jeux de plateau comme War of the Ring (Simulation Publications Inc., 1976) ou La Guerra dell'Anello utilisent des cartes-événements qu'on peut utiliser à certains moments et qui ont des effets sur le jeu semblables aux effets d'un événement spécifique du récit. Ainsi, certaines cartes recréeront l'influence néfaste de Gollum sur les Hobbits. Le récit peut trouver malgré tout un chemin pour s'y immiscer.

21 Ce que cette idée de thème met en évidence, c'est que les conventions d'un genre ne sont pas toujours adaptées à la recréation d'un univers précis. Alors qu'il semblerait, par exemple, que le STR soit un genre très adapté à la recréation d'une guerre, Battle for Middle-Earth montre au contraire plusieurs incohérences. Lors des assauts d'Helm's Deep et de Minas Tirith, événements centraux respectivement des films The Two Towers (Peter Jackson, 2002) et The Return of the King (Peter Jackson, 2003), on constate que les conventions du STR sont peu adaptées au siège d'une forteresse. Dans les deux cas, les assiégeants comme les assiégés ont des bâtiments sur le champ de bataille et accumulent de l'or pour créer de nouvelles unités militaires (Adams, 2004). À Minas Tirith, par exemple, Sauron n'arrive pas avec une armée complète qu'il a pris soigneusement le temps de regrouper sous la même bannière : il doit construire une forteresse au pied de la ville, puis des fournaises pour y accumuler des points de ressources, en sorte de pouvoir créer de nouvelles unités militaires. Le Gondor, de son côté, n’a plus le sentiment de devoir protéger chacun des soldats de sa garnison : il accumule sans limite de l'or grâce aux fermes qui restent actives à l'extérieur comme à l'intérieur de la ville. De nouvelles unités militaires pourront aussi véritablement « apparaître » au sein de la forteresse.

C'est du côté de la culture du modding ${ }^{10}$ qu'on peut retrouver des adaptations de Tolkien plus audacieuses qui semblent chercher à mieux coller à la Terre du Milieu. Ainsi, TW_King_Kong a proposé une version modifiée du jeu Medieval II : Total War (Creative Assembly, 2006) où, contrairement au STR, les sièges permettent de créer un sentiment plus grand de vraisemblance. Ainsi, comme dans les jeux de la série Total War, aucune unité ne peut être " générée » sur le champ de bataille. Le système d'alliances proposé est plus complexe et témoigne un peu mieux de la complexité de l'unification des peuples 
libres contre l'armée de Sauron. Un mod du jeu Crusader Kings II (Paradox Development Studio, 2012), intitulé "CK2: Middle Earth Project», permet de son côté de jouer avec plus de précision dans l'univers à travers le temps, en mettant l'accent sur la succession des souverains et les bris et unifications des royaumes. L'industrie du jeu vidéo a une forte propension à la normalisation des genres vidéoludiques en reprenant des mécaniques de jeu conventionnelles et habituelles, alors que les créateurs de mods semblent plus souvent se permettre une plus grande liberté et audace en termes d'adaptation.

\section{Revivre l'émotion et l'incertitude}

Reiner Knizia, l'auteur du Lord of the Rings Board Game (2000), note bien que c'est davantage l'esprit du récit et les sujets qui y sont abordés que la lettre de celui-ci qui l'intéressait lorsqu'il a créé son jeu (cité dans Salen \& Zimmerman, 2004, p. 25). Lord of the Rings est un récit où l'idée de quête personnelle est centrale, tout en nécessitant une coopération entre les personnages et entre les différentes factions impliquées dans la guerre. C'est un récit où l'ennemi mise sur la peur et la terreur pour conquérir: une émotion véhiculée plus fortement lorsqu'on ne peut pas évaluer rationnellement la force de son adversaire.

Ainsi, les adaptations explorées jusqu'à présent ne prennent pas en compte l'information que le joueur possède. Pour deux raisons, un joueur de n'importe lequel de ces jeux sait tout à fait qu'il a une chance de gagner peu importe le côté qu'il choisira d'incarner - si bien sûr il en a le choix. Comme on l'a déjà précisé, s'il a déjà lu le roman ou vu les films, il sait bien que l'éventuelle destruction de l'anneau est une possibilité très probable. Mais plus encore : un joueur sait qu'un jeu est le plus souvent créé en ayant en tête que chacun des joueurs doit avoir une chance de gagner.

C'est le principe même de la notion d'équilibre dans les jeux - une traduction du terme anglophone balance. Comme Ernest Adams et Andrew Rollings l'écrivent, l'équilibre concerne deux éléments: l'idée que chaque joueur ait une chance équivalente de l'emporter au début de la partie; l'idée que tout au long de la partie, aucun joueur n'ait un avantage indu qui ne puisse être compensé par une action d'un adversaire, sauf avec modération en ce qui concerne la chance (Adams \& Rollings, 2007, p. 368). L'équilibre est en ce sens un objectif de conception de jeu pour que l'expérience des joueurs apparaisse juste. Si l'un des deux joueurs incarne un être démoniaque avec des pouvoirs immenses, comment cette incarnation peut-elle permettre de créer un jeu équilibré ? Plus encore, parce qu'il sait qu'il joue à un jeu, le joueur percevra d'une manière ou d'une autre que chaque faction a sans doute une chance relativement équivalente de l'emporter. Le sentiment de doute du camp des Hobbits et le sentiment de puissance de Sauron n'ont pas énormément de chances de se manifester longtemps.

Comment alors jouer avec une incertitude du résultat final semblable aux protagonistes de l'histoire? Comment recréer, par exemple, le sentiment qu'a Sauron que gagner ses batailles lui permettra de trouver l'anneau ? Comment recréer la difficulté inhérente aux peuples libres de se coordonner? La Guerra dell'Anello prend le pari de le faire, en créant deux objectifs de jeu opérant en parallèle. Le joueur des peuples libres (le Rohan, le Gondor, le Nord, les Elfes et les Nains) affronte le joueur de l'ombre (le Mordor, l'Isengard et les Haradrims \& Orientaux) sur un plateau semblable à Risk. Le joueur des peuples libres doit gérer la politique de chacune des nations impliquées avant de pouvoir les faire 
entrer en guerre. Il doit aussi déplacer la compagnie de l'anneau pour éventuellement le détruire. Le joueur qui incarne Sauron peut gagner avec ses unités militaires, mais doit envoyer ses servants à la traque de l'anneau. Les deux joueurs peuvent gagner par des objectifs militaires, contrairement au récit original où tout se jouera par le destin de l'anneau.

Chaque joueur a un certain nombre de dés d'action qui lui permettent de déplacer des pièces, chercher l'anneau ou le déplacer, recruter des unités ou utiliser une carteévénement. Avant le jet de dés d'action, le joueur de l'ombre peut choisir d'attribuer certains dés à la recherche de l'anneau. Plus il y a de dés consacrés à cette recherche, plus il est difficile pour la communauté de l'anneau de se déplacer sans subir de points de corruption. Le déplacement de la communauté reste caché : dans les faits, tant que la communauté n'est pas révélée par les règles du jeu, ${ }^{11}$ son pion reste au même endroit tout en accumulant des points de déplacement. La communauté utilisera ses points de déplacements lorsqu'elle est découverte ou lorsqu'elle se révèle d'elle-même, pouvant dans ce dernier cas arrêter dans des forteresses alliées pour soigner sa corruption. Les compagnons de l'anneau peuvent rester avec la compagnie et sacrifier leurs vies pour empêcher l'accumulation de points de corruption, ou encore quitter la compagnie et aider au côté militaire - risquant alors de tomber au combat. Le joueur de l'ombre gagnera si les porteurs de l'anneau accumulent trop de points de corruption ; par contre, si le joueur des peuples libres emmène l'anneau jusqu'à la Montagne du Destin, c'est lui qui gagne.

Parallèlement, les deux joueurs peuvent gagner avec des objectifs militaires. Étrangement, le joueur des peuples libres peut gagner en accumulant quatre points d'objectifs militaires, ${ }^{12}$ alors que le joueur de l'ombre doit en accumuler dix. Le manuel d'instructions du jeu clarifie les raisons diégétiques de ces objectifs :

Si Sauron avait réussi à anéantir les Nations des Peuples Libres, même la destruction de l'Anneau n'aurait pas abouti à une véritable victoire pour ceux-ci. D'un autre côté, si les Peuples Libres avaient donné encore plus de fil à retordre au Seigneur des Ténèbres sur le champ de bataille, celui-ci aurait été si concentré sur le conflit qu'il aurait été bien plus facile aux Porteurs de l'Anneau de s'infiltrer dans les entrailles du Mordor ${ }^{13}$.

Le joueur des peuples libres peut, dans les faits, prendre la Moria et l'Isengard - tout deux très loin de la Montagne du Destin - et gagner la partie. La facilité qu'auraient les porteurs de l'anneau de s'infiltrer par la suite au Mordor est certainement à nuancer.

Mais la raison de cet écart entre les points est plutôt ludique que narrative : il est plus facile pour le joueur de l'ombre de prendre des forteresses que pour le joueur des peuples libres, en raison de sa supériorité numérique. Si le joueur de l'ombre se consacre uniquement à l'anneau, le sentiment inverse peut alors se développer: il semble plus facile pour le joueur des peuples libres d'atteindre une victoire militaire. Cet objectif militaire force en partie le joueur de l'ombre à lancer rapidement plusieurs assauts, rendant son attaque plus longue et plus soutenue tout au long de la partie. D'un autre côté, il force le joueur de l'ombre à attaquer et à se défendre sur le plan militaire : on peut imaginer que sans cet objectif, Sauron pourrait décider de mettre tous ses efforts à la recherche de l'anneau, négligeant alors ses armées qui sont pourtant à la source du sentiment de puissance et de l'effet de terreur propre au récit source. Comme le joueur qui incarne Sauron sait que l'anneau a une chance non-négligeable d'être détruit, il pourrait décider de changer complètement la stratégie originale de son personnage pour 
se consacrer à retrouver l'objet de sa convoitise. Dès lors, pour que l'incarnation de Sauron soit similaire d'un média à l'autre, le jeu de société a choisi d'intégrer des mécaniques de jeu qui l'incitent à avoir au minimum suffisamment d'armées pour défendre ses forteresses. Puisque les deux joueurs doivent constamment mobiliser leurs efforts sur deux fronts, ils placent leurs espoirs dans deux objectifs parallèles ; un joueur qui abandonne l'un des deux objectifs laisse le champ à l'autre d'y dominer. Ainsi, même si le contexte diégétique doit être adapté, l'attention des deux joueurs est divisée sur deux fronts, permettant de recréer un sentiment d'incertitude analogue au sentiment des personnages présents dans le monde de Tolkien.

31 Le jeu traditionnel semble ici moins subordonné aux conventions de genre au bénéfice de l'expérience renouvelée des enjeux de la guerre de l'anneau. C'est que, contrairement au jeu vidéo, l'expérience d'un jeu de société a une constituante fondamentale qui y rend moins nécessaire une «lecture préfabriquée»: les joueurs doivent lire le manuel d'instructions. Plutôt que d'avoir une expérience basée sur les conventions de genre, les habitudes stratégiques et l'implémentation des mécaniques dans un logiciel, le jeu traditionnel mise sur l'apprentissage à partir de la lecture et de l'application manuelle des règles par les joueurs.

Cette opposition entre la lecture d'un manuel d'instructions d'une part et les conventions de genre de l'autre n'est par contre pas liée aux deux médias d'une manière ontologique. Non seulement Risk: The Lord of the Rings permet de jouer avec les règles du Risk original, mais les instructions tiennent compte de l'expérience du premier jeu : pour les joueurs habitués aux règles de Risk, on peut abréger la lecture du manuel en s'en tenant aux parties du texte clairement identifiées en bleu. Dans le même esprit, les règles du Monopoly (Charles Darrow, 1933) sont assez connues pour n'être à peu près jamais lues, sans toutefois qu'elles ne soient véritablement respectées à la lettre. ${ }^{14}$ À l'inverse, les jeux vidéo ont aussi nécessité longtemps la lecture de manuels d'instructions. Chris Lombardi (1995, p. 228), dans une critique de Warcraft: Orcs \& Humans (Blizzard Entertainment, 1994), note que le jeu ressemble tellement à Dune II : The Building of A Dynasty (Westwood Studios, 1992) qu'un joueur peut probablement se rendre jusqu'à la moitié du jeu sans même consulter le manuel. Cet indice montre l'évidence qu'a cette lecture pour quelqu'un qui souhaite apprendre un jeu vidéo en 1995. Pour questionner la notion de genre et son importance dans l'étude des jeux, on devra certainement se pencher sur les habitudes préalables et le rôle du manuel pour mieux comprendre l'équilibre entre convention et originalité dans l'histoire du jeu.

\section{Conclusion}

Cette réflexion nous aura permis un tour d'horizon de différents enjeux transmédiatiques : l'adaptation d'un récit - littéraire d'abord puis cinématographique en jeu vidéo et en jeu de société entraîne des questions variées liées à la posture du joueur. Pour rejouer l'histoire, certains jeux vont simplement la raconter à nouveau à la manière du cinéma ou de la littérature en limitant les possibilités d'action. Pour s'immerger dans l'univers même, d'autres jeux prennent le monde comme un thème en $y$ superposant des mécaniques de jeu très centrées sur des conventions de genres qui a priori n'y ont pas grand-chose à voir. Enfin, un plus petit nombre de jeux - en particulier des jeux traditionnels, mais aussi des mods - vont chercher à recréer un sentiment plus ou 
moins semblable à celui des personnages, adaptant pour cela l'esprit du récit et le sentiment des personnages eux-mêmes.

Ces questions s'inscrivent dans une réflexion qui met en avant la jouabilité elle-même, largement négligée à mon sens dans l'étude des jeux vidéo. Comme le rappelait déjà Espen Aarseth en 2003, un jeu est un processus et non un objet (Aarseth, 2003, p. 2); le chercheur qui veut comprendre l'expérience d'un joueur doit en comprendre le contexte. Si en faire l'expérience constitue très certainement le point de départ de toute analyse détaillée d'un jeu, analyser la jouabilité en contexte s'avère plus complexe: il faut pour cela mieux comprendre les usages typiques d'une période donnée, d'une communauté ou d'un mode de jeu, selon les champs d'intérêt. Comprendre le genre non pas uniquement comme modèle de création d'un jeu, mais plus spécifiquement comme stratégie interprétative de joueurs : voilà la posture que ce texte a cherché à mettre en évidence. L'expérience réelle et avérée de joueurs peut faire fi de la fidélité à l'œuvre originale en y retrouvant des points d'intérêts qui, parfois, rejoignent «l'esprit» de celle-ci. C'est en tenant en compte du rôle fondamental de la jouabilité et de la posture du joueur qu'on pourra mieux comprendre de manière générale tout ce que la culture ludique implique.

\section{BIBLIOGRAPHIE}

AARSETH E. (2003), « Playing research: methodological approaches to game analysis », Melbourne DAC, the 5th International Digital Arts and Culture Conference, Melbourne, http:// hypertext.rmit.edu.au/dac/papers/Aarseth.pdf

ADAMS D. (2004), « The Lord of the Rings: Battle for Middle-earth », IGN, 3 décembre, http:// ca.ign.com/articles/2004/12/04/the-lord-of-the-rings-battle-for-middle-earth

ADAMS E. \& ROLLINGS A. (2007), Fundamentals of game design, Englewood Cliffs, Prentice Hall.

BIZZOCCHI J. \& TANENBAUM J. (2011), « Well Read: Applying Close Reading Techniques to Gameplay Experiences », In Davidson D. (dir.), Well Played 3.0: Video Games, Value and Meaning, http://www.etc.cmu.edu/etcpress/content/well-played-30-video-games-value-and-meaning

BLANCHET A. (2008), « Des films aux jeux vidéo : quand le jeu impose ses règles », In Brougère, G., La ronde des jeux et des jouets. Harry, Pikachu, Superman et les autres, Paris, Autrement, pp. 59-75.

CISNEROS J. R. (2000), Technologies of reception: on the adaptation of Latin American literature to the cinema, thèse de doctorat, Université de Montréal.

DOR S. (2014), « The Heuristic Circle of Real-Time Strategy Process : A StarCraft : Brood War Case Study ». Game Studies, vol. 14, n 1, http://gamestudies.org/1401/articles/dor

ECO U. ([1979] 1985), Lector in fabula, Le rôle du lecteur ou la Coopération interprétative dans les textes narratifs, Paris, Grasset.

GAUDREAULT A. (1999), Du littéraire au filmique : système du récit, Québec/Paris, Nota bene/ Armand Colin. 
GENSERIC (s.d.), « Test Le Seigneur des Anneaux : La Bataille pour la Terre du Milieu [BFME] », JeuxVideoPC, http://www.jeuxvideopc.com/test/7915-le-seigneur-des-anneaux-la-bataille-pourla-terre-du-milieu.php

HOLDING D. H. (1985), The psychology of chess skill, Hillsdale, L. Erlbaum Assoc.

JENKINS H. ([2002] 2004), « Game Design as Narrative Architecture », Electronic Book Review, http://www.electronicbookreview.com/thread/firstperson/lazzi-fair. D'abord paru In Harrington P. \& Frup-Waldrop N. (dir.), First Person, Cambridge MA, MIT Press.

JOE_DODSON (2004), « The Lord of the Rings: Battle for Middle Earth Review », GameRevolution, 12 janvier, http://www.gamerevolution.com/review/the-lord-of-the-rings-battle-for-middle-earth JUUL J. (2005), Half-real : video games between real rules and fictional worlds, Cambridge MA, MIT Press.

KEEFER J. (2004), « The Lord of the Rings: The Battle for Middle-earth », GameSpy, http:// pc.gamespy.com/pc/the-lord-of-the-rings-the-battle-for-middle-earth/570709p1.html

KLASTRUP L. \& TOSCA S. (2004), « Transmedial worlds - rethinking cyberworld design », In Nakajima M., Hatori Y. \& Sourin A. (dir.), Actes du colloque International Conference on Cyberworlds 2004, pp. 409-416.

LESSARD J. (2013), Histoire formelle du jeu d'aventure sur ordinateur (le cas de l'Amérique du Nord de 1976-1999), thèse de doctorat, Université de Montréal.

LOMBARDI C. (1995), « War Crime in Real Time », Computer Gaming World, n 126, pp. 228-232.

OCAMPO J. (2004), « The Lord of the Rings, The Battle for Middle-earth Review », Gamespot, 7 décembre, http://www.gamespot.com/reviews/the-lord-of-the-rings-the-battle-for-middleearth-/1900-6114604/

PEIRCE C. S. (1991), « Some Consequences of Four Incapacities », In Peirce on signs : writings on semiotic, Chapel Hill, University of North Carolina Press, pp. 54-84.

RABINOWITZ P. J. (1987), Before reading : narrative conventions and the politics of interpretation, Ithaca, Cornell University Press.

RYAN M.-L. (2001), « Beyond Myth and Metaphor - The Case of Narrative in Digital Media », Game Studies, vol. 1, n 1, http://www.gamestudies.org/0101/ryan/

SALEN K. \& ZIMMERMAN E. (2004), Rules of play : game design fundamentals, Cambridge, MIT Press. SUPERPANDA (2004), « Test de Le Seigneur des Anneaux : La Bataille pour la Terre du Milieu sur PC », JeuxVideo.com, 9 décembre, http://www.jeuxvideo.com/articles/0000/00004915_test.htm

TUCKER R. (s.d.), « Lord of the Rings: Battle for Middle-earth », Game Vortex, http:// www.gamevortex.com/gamevortex/soft_rev.php/2409/lord-of-the-rings-battle-for-middleearthpc.html

WALLIN M. R. (2007), « Myths, monsters and markets: ethos, identification, and the video game adaptations of The Lord of the Rings ", Game Studies, vol. 7, n 1, http://gamestudies.org/0701/ articles/wallin

\section{NOTES}

1. Les citations en anglais sont traduites par l'auteur. 
2. Le cas des suites ou des franchises au cinéma ou en littérature pose aussi ce type de problème. Le spectateur qui, par exemple, souhaite voir les films de Star Wars incluant les prequels a nécessairement intérêt à ne pas les voir dans l'ordre chronologique des événements de la fiction (de I à, éventuellement, IX), mais plutôt dans leur ordre de sortie s'il souhaite vivre la surprise de The Empire Strikes Back (Irvin Kershner, 1980).

3. L'expression hack and slash, parfois traduite par " porte-monstre-trésor », renvoie à l'idée d'un jeu où l'expérience est essentiellement d'explorer un souterrain, d'éliminer les ennemis qui s'y trouvent et de collecter les trésors et points d'expérience qui en résultent pour améliorer ses personnages.

4. Notons par contre que ce ne sont pas tous les STR qui ont des héros, ni qui permettent à ceuxci d'accumuler des points d'expérience.

5. C'est aussi le cas dans le jeu sur Gameboy Advance The Lord of the Rings: The Third Age (Griptonite Games, 2004), qui ne doit pas être confondu avec le jeu dont on parlera plus bas (qui porte le même nom et est sorti la même année). Le jeu propose des mécaniques très semblables à la série Fire Emblem. Le joueur peut activer le mode "Sauron ", ajoutant une difficulté plus élevée en ne permettant pas aux héros morts au combat d'être ressuscités.

6. Pour une raison étonnante, par contre, Battle for Middle-Earth adapte l'histoire originale en permettant à Boromir de revenir pour les scénarios qui se déroulent au Gondor, même s'il meurt bel et bien dans l'histoire originale. Gandalf doit aussi gagner son combat contre le Balrog et accompagnera la compagnie pour le reste du jeu, même si sa défaite et son retour sont des événements centraux au récit.

7. J'ai développé plus longuement la notion d'habitude stratégique dans un précédent article (Dor, 2014).

8. Je dois à Dominic Arsenault la découverte de ce jeu et l'observation de son étrangeté dans une conférence intitulée «Lord of the Rings: The Third Age : Comment briser un univers de 50 ans en 5 moments / 5 minutes » présentée le 31 août 2011 dans le cadre des rencontres du Reliquaire.

9. Des variantes officielles incluent la possibilité d'alliances ou d'équipes entre les armées du bien et du mal. Il reste que la complexité de la relation entre Sauron et l'Isengard, par exemple, n'y est pas.

10. Le modding est l'action de modifier un logiciel de jeu vidéo - avec ou sans l'aval des développeurs et éditeurs - pour proposer certaines variantes systémiques ou cosmétiques ou pour améliorer l'expérience originale.

11. Chaque dé mis à la traque de l'anneau est un dé traditionnel (à six faces) pour trouver l'anneau: pour chaque lancer de six, le joueur pige une tuile parmi une pioche qui ajoutera un certain nombre de points de corruption aux porteurs de l'anneau et parfois le forcera à se révéler. Si le joueur se déplace à nouveau dans le tour, c'est au lancer de cinq ou six qu'aura lieu la pige, et ainsi de suite.

12. Une forteresse équivaut à deux points alors qu'un établissement régulier, un point.

13. Roberto Di Meglio, Marco Maggi et Francesco Nepitello ([2004] 2005), La guerre de l'anneau. Règles complètes. Édition révisée, Ares Games (p. 22).

14. On peut penser bien sûr ici à la règle du «stationnement gratuit ", où un joueur qui tombe sur la case du même nom récolte le montant d'argent collecté par les cartes « Chance » et «Caisse commune ». Cette règle très commune n'a pourtant aucune existence dans les instructions officielles. 


\section{RÉSUMÉS}

L'univers de J.R.R. Tolkien semble avoir une propension à susciter un fort sentiment d'immersion dans différents genres ludiques. Mais la connaissance préalable d'un récit mène à une expérience bien différente de celle d'une histoire inconnue. À travers l'exploration de différentes adaptations vidéoludiques, notamment le jeu de stratégie en temps réel The Lord of the Rings: The Battle for Middle-earth (EA Los Angeles, 2004), on verra comment celles-ci tendent à vouloir préserver des mécaniques de genres conventionnelles. Au contraire, le jeu de plateau La Guerra dell'Anello (Roberto Di Meglio, Marco Maggi et Francesco Nepitello, 2004) en adapte l'esprit plutôt que la lettre, en se centrant d'une manière plus précise sur le sentiment d'incertitude des personnages. Cet article propose une analyse comparative de différentes stratégies d'adaptation, entre jeux traditionnels et jeux numériques, dans un contexte où un joueur ou une joueuse en connaît déjà les enjeux narratifs majeurs.

J.R.R. Tolkien's Middle-earth is one of the most adapted universes in games from various genres. But the prior knowledge of a narrative leads to a whole different experience than when one's faced with an unknown story. This article will deal with different videogames adaptation, including the real-time strategy game The Lord of the Rings: The Battle for Middle-earth (EA Los Angeles, 2004), to show how they tend to stay within conventional genres. The board game La Guerra dell'Anello (Roberto Di Meglio, Marco Maggi et Francesco Nepitello, 2004), on the contrary, adapts the spirit rather than the letter of the story, focusing on the uncertainty shared by the characters towards their own objectives. This paper is essentially a comparative analysis of different adaptation strategies, from traditional games to digital games, in a context where the player knows beforehand major plot points.

INDEX

Keywords : digital games, board game, genres, adaptation, narration, J.R.R. Tolkien

Mots-clés : jeu numérique, jeu de société, genres, adaptation, narration, J.R.R. Tolkien

\section{AUTEUR}

SIMON DOR

Université de Montréal 